Proceedings of the National Academy of Sciences

Vol. 67, No. 1, pp. 282-289, September 1970

\title{
Inference from Inadequate and Inaccurate Data, III*
}

\author{
George Backus
}

UNIVERSITY OF CALIFORNIA, LA JOLLA, CALIF. 92037

Communicated June 12, 1970

\begin{abstract}
Having measured $D$ numerical properties of a physical object $E$ which requires many more than $D$ parameters for its complete description, we want to estimate $P$ other numerical properties of $E$. Continuing the discussion in papers $\mathrm{I}^{1}$ and $\mathrm{II},{ }^{2}$ the present paper gives estimates when we believe it likely that we can guess an upper bound $M$ on the Hilbert norm not of $\boldsymbol{h}_{E}$, the model representing $E$ in some Hilbert space, but of the orthogonal projection of $\boldsymbol{h}_{E}$ onto a sufficiently large subspace. In addition, the present paper simplifies the notation of I, and makes explicit the application of Bayesian subjective probability when there are errors in the data and we want to find the joint probability distribution of more than one prediction.
\end{abstract}

1. Notation. Old German capitals will stand for closed subspaces of real Hilbert spaces, a capital script letter will denote the orthogonal projection operator ${ }^{3}$ from the whole Hilbert space onto the subspace named by the same letter in old German, and bold-face lower-case italics will denote vectors in Hilbert spaces. The inner product of $f$ and $g$ will be written $\langle f, g\rangle$ and also $f \cdot g ;\|f\|$ will denote $\langle f, f\rangle^{1 / 2}$. If $V: \mathfrak{S} \rightarrow \mathfrak{S}$ is a bounded linear operator sending the Hilbert space $\mathfrak{S}$ into itself, $V \cdot f$ will denote the vector obtained by applying $V$ to $f$. If $V^{*}$ is the adjoint $^{3}$ of $V$, then $V^{*} \cdot f$ will sometimes be written $f \cdot V$, so that $(f \cdot V) \cdot g=f$. $(\boldsymbol{V} \cdot \boldsymbol{g})$. If $\boldsymbol{W}: \mathfrak{S} \rightarrow \mathfrak{S}$ is another bounded linear operator then $V \cdot W: \mathfrak{S} \rightarrow \mathfrak{S}$ will be defined by requiring $(\boldsymbol{V} \cdot W) \cdot f=V \cdot(\boldsymbol{W} \cdot \boldsymbol{f})$ for all $f$ in $\mathfrak{S}$. If $S$ is any subset of $\mathfrak{H}, \boldsymbol{V} \cdot \boldsymbol{S}$ will denote the set of all vectors in $\mathfrak{S}$ which have the form $\boldsymbol{V} \cdot \boldsymbol{s}$ for some $\boldsymbol{s}$ in $S$. The set whose members are $f_{1}, \ldots, f_{q}$ will be written $\left\{f_{1}, \ldots, f_{q}\right\}$. If $P(\boldsymbol{h})$ is any statement about the arbitrary vector $\boldsymbol{h}$ in $\mathfrak{S}$ then $\{\boldsymbol{h}: P(\boldsymbol{h})\}$ will denote the set of all $\boldsymbol{h}$ in $\mathfrak{S}$ for which $P(\boldsymbol{h})$ is true.

If $\Re$ and $\subseteq$ are closed subspaces of Hilbert space $\mathfrak{S}$ and $\subseteq \subseteq \Re$ then $\Re \ominus \subseteq$ will denote the set of all vectors in $\Re$ which are orthogonal to every vector in $\subseteq$. The space $\mathfrak{S} \ominus \Re$ will be written $\Re^{\perp}$, and $\mathfrak{R}: \mathfrak{S} \rightarrow \Re$ and $\mathfrak{R}^{\perp}: \mathfrak{S} \rightarrow \Re^{\perp}$ will denote the orthogonal projection operators from $\mathfrak{S}$ onto $\Re$ and $\mathfrak{R}^{\perp}$. The identity operator on $\mathfrak{S}$ will be written $\mathfrak{H}$.

If $V: \mathfrak{S} \rightarrow \mathfrak{S}$ is a bounded linear operator and $\Re$ is a closed subspace of $\mathfrak{S}$, then $V \mid \Re$ will denote the operator $\tilde{V}: \Re \rightarrow \mathfrak{S}$ defined on $\Re$ by requiring that $\tilde{V} \cdot \boldsymbol{r}=\boldsymbol{V} \cdot \boldsymbol{r}$ for every $\boldsymbol{r}$ in $\Re$. If $\boldsymbol{V} \cdot \Re \subseteq \Re$, and $V \cdot \Re^{\perp}=\{\boldsymbol{o}\}$, and $(V \mid \Re)^{-1}: \Re \rightarrow \Re$ exists and is bounded, we will say that $V$ lives on $\Re$, and we will define $V^{\text {inv }}: \mathfrak{S} \rightarrow \mathfrak{S}$ as $V^{\text {inv }}=(V \mid \Re)^{-1} \cdot \Re$. Then $V^{\text {inv }}$ is the generalized inverse of $V$ defined by Moore ${ }^{4}$ and Penrose, ${ }^{5}$ and is the only linear mapping $U: \mathfrak{F} \rightarrow \mathfrak{S}$ such that $U \cdot \dot{V}=V \cdot U=\mathscr{R}$ and $U \cdot \mathbb{R}^{\perp}=O$. We note that $V^{*}$ and $V^{\text {inv }}$ also 
live on $\Re$ and $\left(\boldsymbol{V}^{\text {inv }}\right)^{\text {inv }}=\boldsymbol{V},\left(\boldsymbol{V}^{*}\right)^{\text {inv }}=\left(\boldsymbol{V}^{\text {inv }}\right)^{*}$. Also, if $\boldsymbol{V}$ and $\boldsymbol{W}$ both live on $\Re$ then so does $V \cdot W$, and $(V \cdot W)^{\text {inv }}=W^{\text {inv }} \cdot V^{\text {inv }}$.

If $\mathfrak{S}$ and $\mathfrak{T}$ are two subspaces of $\mathfrak{S}, \mathfrak{S}+\mathfrak{T}$ will denote the subspace consisting of all vectors $s+t$ with $s$ in $\mathfrak{S}$ and $t$ in $\mathfrak{T}$. If $\mathfrak{S}$ and $\mathfrak{T}$ are mutually orthogonal, $\mathfrak{S}+\mathfrak{T}$ will be written $\mathfrak{S} \oplus \mathfrak{T}$. The angle between $\mathfrak{S}$ and $\mathfrak{T}, \angle(\mathfrak{S}, \mathfrak{T})$, will be defined as the infimum of the positive angles between pairs of vectors $(s, t)$ with $s$ in $\subseteq$ and $t$ in $\mathfrak{T}$. The angle between $O$ and any vector is taken to be $\pi / 2$. Clearly if $\mathfrak{S} \cap \mathfrak{T} \neq\{0\}$ then $\angle(\mathfrak{S}, \mathfrak{T})=0$; the converse is false. ${ }^{6}$

2. Oblique Subspaces. If $\subseteq$ and $\mathfrak{T}$ are subspaces of Hilbert space $\mathfrak{T}$ such that $\mathfrak{S} \cap \mathfrak{T}=\{0\}$, we can define two linear operators, $\mathfrak{S}_{\mathfrak{J}}: \mathfrak{S}+\mathfrak{T} \rightarrow \mathfrak{S}$ and $\mathfrak{J}_{\mathfrak{S}}: \mathfrak{S}+$ $\mathfrak{I} \rightarrow \mathfrak{I}$, by requiring that for any $s$ in $\mathfrak{S}$ and any $t$ in $\mathfrak{T}$ we have $\mathcal{S}_{\mathfrak{J}} \cdot(s+t)=s$ and $J_{\mathfrak{S}} \cdot(\mathfrak{s}+t)=t$. We call $\mathfrak{S}_{\mathfrak{J}}$ the projection of $\mathfrak{S}+\mathfrak{T}$ onto $\mathfrak{S}$ parallel to $\mathfrak{T}$. Evidently $S_{\mathfrak{J}} \mid \mathfrak{S}$ is the identity operator on $\mathfrak{S}$ and $\delta_{\mathfrak{J}} \mid \mathfrak{T}$ is the $\boldsymbol{o}$ operator on $\mathfrak{T}$, and $\mathfrak{S}_{\mathfrak{J}}+\mathfrak{J}_{\mathfrak{S}}$ is the identity operator on $\mathfrak{S}+\mathfrak{I}$.

Lorch $^{6}$ has used the closed graph theorem ${ }^{7}$ to show that when $\subseteq$ and $\mathfrak{I}$ are closed and $\mathfrak{S} \cap \mathfrak{T}=\{0\}$ the following three statements are equivalent: (i) $\mathfrak{S}+\mathfrak{I}$ is closed; (ii) $\angle(\mathfrak{S}, \mathfrak{T})>0$; (iii) $\mathfrak{S}_{\mathfrak{J}}$ is bounded. He has also shown ${ }^{6}$ that these statements are always true if $\mathfrak{S}$ or $\mathfrak{T}$ is finite-dimensional.

If $\mathfrak{S}$ and $\mathfrak{T}$ are closed subspaces of Hilbert space $\mathfrak{T}$ such that $\mathfrak{S} . \cap \mathfrak{T}=\{0\}$ and $\mathfrak{S}=\mathfrak{S}+\mathfrak{T}$, we write $\mathfrak{S}=\mathfrak{S}+\mathfrak{T}$. Lorch's remark shows that if $\mathfrak{S}=$ $\mathfrak{S}+\mathfrak{I}$ then $\varsigma_{\mathfrak{J}}: \mathfrak{S} \rightarrow \mathfrak{S}$ and $\mathfrak{J}_{\mathfrak{S}}: \mathfrak{S} \rightarrow \mathfrak{T}$ are bounded. Moreover, $\varsigma_{\mathfrak{J}}+\mathfrak{J}_{\mathfrak{S}}=\mathfrak{T}$, $S_{\mathfrak{J}}{ }^{2}=S_{\mathfrak{J}}$, and $S_{\mathfrak{J}} \cdot \mathfrak{J}_{\mathfrak{S}}=0$.

We also have

$$
\begin{aligned}
& \delta_{\mathfrak{J}} \cdot s=s, \quad s \cdot s_{\mathfrak{J}}=\delta_{\mathfrak{J}}, \\
& S_{\mathfrak{J}} \cdot \mathfrak{J}=0, \quad J \cdot S_{\mathfrak{J}}=\mathfrak{J}-J_{\mathfrak{S}}, \\
& S_{\mathfrak{J}} \cdot S^{\perp}=S_{\mathfrak{J}}-S, \quad \quad S^{\perp} \cdot S_{\mathfrak{J}}=0, \\
& \delta_{\mathfrak{J}} \cdot \mathfrak{J}^{\perp}=\delta_{\mathfrak{J}}, \quad \mathfrak{J}^{\perp} \cdot \delta_{\mathfrak{J}}=\mathfrak{J}^{\perp} .
\end{aligned}
$$

Formulae (1), $(2 a)$ and (3b) are obvious and the others follow from them and the identities $S+S^{\perp}=\mathfrak{J}+\mathfrak{J}^{\perp}=S_{\mathfrak{J}}+J_{\mathfrak{S}}=\mathfrak{H}$.

If $\mathfrak{S}=\mathfrak{S}+\mathfrak{T}$, a simple calculation which we omit shows that $\mathfrak{S}=\mathfrak{S}^{\perp} \dot{+} \mathfrak{T}^{\perp}$ and $S_{J^{*}}{ }^{*} \mathfrak{J}^{\perp} s^{\perp}$.

Now suppose that $\mathfrak{S}=\mathfrak{S}+\mathfrak{T}$, that $A: \mathfrak{S} \rightarrow \mathfrak{S}$ lives on $\mathfrak{S}$, that $B: \mathfrak{F} \rightarrow \mathfrak{T}$ lives on $\mathfrak{I}$, and that $Q: \mathfrak{T} \rightarrow \mathfrak{S}$ is linear: Then an easy calculation, omitted here, shows that $\boldsymbol{U}=\boldsymbol{A}+\boldsymbol{S} \cdot \boldsymbol{Q} \cdot \mathfrak{J}+\boldsymbol{B}$ has a bounded inverse on $\mathfrak{S}$ and that

$$
U^{-1}=S_{\mathfrak{J}}^{*} \cdot A^{\text {inv }} \cdot S_{\mathfrak{J}}-S_{\mathfrak{J}}{ }^{*} \cdot A^{\text {inv }} \cdot Q \cdot B^{\text {inv }} \cdot J_{\mathfrak{S}}+\mathfrak{J}_{\mathfrak{S}}^{*} \cdot B^{\text {inv }} \cdot J_{\mathfrak{S}} \cdot
$$

When $\mathfrak{S}=\mathfrak{S}+\mathfrak{T}$, there is a useful variational characterization of $\varsigma_{\mathfrak{J}}$. Let $\mathfrak{U}$ be any closed subspace of $\mathfrak{S}$ such that $\mathfrak{U}=(\mathfrak{S} \cap \mathfrak{U}) \oplus \mathfrak{T}$. Then for any $\boldsymbol{h}$ in $\mathfrak{S}, \boldsymbol{S}_{\mathfrak{J}} \cdot \boldsymbol{h}$ is that vector $\boldsymbol{s}$ in $\mathfrak{S} \cap(\boldsymbol{h}+\mathfrak{u})$ which minimizes $\|\boldsymbol{s}-\boldsymbol{h}\|$. To see this, note first that $S_{\mathfrak{J}} \cdot \boldsymbol{h}=\boldsymbol{h}-\mathfrak{J}_{\mathfrak{J}} \cdot \boldsymbol{h}$, so $\S_{\mathfrak{J}} \cdot \boldsymbol{h}$ is in $\subseteq \cap(\boldsymbol{h}+\mathfrak{u})$. If $\boldsymbol{s}$ is in $\mathfrak{S} \cap$ $(\boldsymbol{h}+\mathfrak{u})$ then $\boldsymbol{s}-s_{\mathfrak{J}} \cdot \boldsymbol{h}$ is in $\mathfrak{S} \cap \mathfrak{U}$ and hence orthogonal to $\mathfrak{T}$. Therefore $\|s-h\|^{2}=\left\|s-\delta_{\mathfrak{J}} \cdot \boldsymbol{h}-\mathfrak{J}_{\mathfrak{J}} \cdot \boldsymbol{h}\right\|^{2}=\left\|s-\delta_{\mathfrak{J}} \cdot \boldsymbol{h}\right\|^{2}+\left\|\delta_{\mathfrak{J}} \cdot \boldsymbol{h}-\boldsymbol{h}\right\|^{2}$, and $\|s-\boldsymbol{h}\|$ $>\left\|s_{\mathfrak{J}} \cdot \boldsymbol{h}-\boldsymbol{h}\right\|$ unless $\boldsymbol{s}=\mathfrak{S}_{\mathfrak{J}} \cdot \boldsymbol{h}$. 
3. Cylinder Measures. If $B$ is a Borel ${ }^{9}$ subset of finite-dimensional closed subspace $\mathfrak{T}$ of Hilbert space $\mathfrak{S}$, then $\mathfrak{J}^{-1}(B)$, the set of all $\boldsymbol{h}$ in $\mathfrak{S}$ such that $\mathfrak{J} \cdot \boldsymbol{h}$ is in $B$, is called a cylinder set ${ }^{8}$ based on $B$. The class $c(\mathfrak{T})$ of all cylinder sets based on Borel subsets of $\mathfrak{T}$ is a $\sigma$-ring, ${ }^{9}$ and the class of all cylinder sets in $\mathfrak{T}$ is a ring. ${ }^{8}{ }^{810}$ A nonnegative real-valued set function $\mu$ defined on all cylinder sets in $\mathfrak{S}$ is called a cylinder measure ${ }^{8,10}$ on $\mathfrak{S}$ if $\mu(\mathfrak{S})=1$ and if $\mu$ is countably additive ${ }^{9}$ on $c(\mathfrak{T})$ for each finite-dimensional subspace $\mathfrak{T}$ of $\mathfrak{S}$. From the definition, $\mu$ is finitely additive on all the cylinder sets of $\mathfrak{S}$, but $\mu$ need not be countably additive there. ${ }^{8}$ On $c(\mathfrak{T}), \mu$ is a probability measure, ${ }^{9}$ which will be written $\mu_{\mathfrak{J}}$ and called the marginal distribution of $\mu$ on $\mathfrak{T}$, by analogy with probability theory on finitedimensional spaces. ${ }^{11}$ The value of $\mu\left(J^{-1}(B)\right)$ will be called the probability that $\mathfrak{J} \cdot \boldsymbol{h}$ is in $B$ if $\boldsymbol{h}$ is in $\mathfrak{S}$.

A function $f: \mathfrak{S} \rightarrow F$ mapping $\mathfrak{S}$ into an arbitrary set $F$ is called a cylinder function based on the finite-dimensional subspace $\mathfrak{T}$ of $\mathfrak{S}$ if, for every $\boldsymbol{h}$ in $\mathfrak{S}$, $f(\boldsymbol{h})=f(\mathfrak{J} \cdot \boldsymbol{h})$. If $f$ is a real-valued, vector-valued or tensor-valued cylinder function based on $\mathfrak{T}$ then the integral of $f$ over $\mathfrak{S}$ with respect to the cylinder measure $\mu$ is defined as

$$
\int_{\mathfrak{W}} f(x) \mu(d x)=\int_{\mathfrak{T}} f(t) \mu_{\mathfrak{J}}(d t)
$$

where the integral on the right is the ordinary integral ${ }^{9}$ over $\mathfrak{T}$ with respect to the probability measure $\mu_{\mathcal{J}}$.

Suppose that $\mu$ is a cylinder measure on $\mathfrak{S}$ and that the linear functional $f: \mathfrak{S}$ $\rightarrow R$ ( $R$ is the real line) defined by $f(\boldsymbol{h})=\int_{\mathfrak{G}} \boldsymbol{h} \cdot \boldsymbol{x \mu}(d x)$ exists and is bounded. Then the unique vector ${ }^{3} \boldsymbol{m}_{\mathfrak{H C}}$ in $\mathfrak{S}$ such that $\boldsymbol{f}(\boldsymbol{h})=\boldsymbol{m}_{\mathfrak{H C}} \cdot \boldsymbol{h}$ for all $\boldsymbol{h}$ in $\mathfrak{S}$ is called the center of mass, or mean, of $\mu$, and is written heuristically as $m_{\mathfrak{H C}}=$ $\int_{\mathfrak{S}} x_{\mu}(d x)$. If $\mathfrak{T}$ is any finite-dimensional subspace of $\mathfrak{S}$ such that $\int_{\mathfrak{T}} t \mu_{\mathfrak{T}^{-}}$ $(d t)=m_{\mathfrak{J}}$ exists, then $m_{\mathfrak{J}}$ is the ordinary mean ${ }^{11}$ of $\mu_{\mathfrak{J}}$ on $\mathfrak{T}$. If the mean $m_{\mathfrak{T}}$ of $\mu$ on $\mathfrak{S}$ exists, then for any finite-dimensional subspace $\mathfrak{T}$ of $\mathfrak{S}$, the mean $\boldsymbol{m}_{\mathfrak{J}}$ exists and equals $\mathfrak{J} \cdot \boldsymbol{m}_{\mathfrak{K}}$.

Suppose that $\mu$ is a cylinder measure on $\mathfrak{S}$ with mean $m_{\mathfrak{K}}$ and that the bilinear functional $f(h, k)=\int_{\mathfrak{g}}\left[h \cdot\left(x-m_{\mathfrak{K}}\right)\right]\left[\left(x-m_{\mathfrak{K}}\right) \cdot \boldsymbol{k}\right] \mu(d x)$ exists and is bounded. It is symmetric and positive semi-definite, so there exists ${ }^{3}$ a unique self-adjoint, positive semi-definite linear operator $V_{\mathfrak{K C}}: \mathfrak{S} \rightarrow \mathfrak{S}$ such that for every $\boldsymbol{h}$ and $\boldsymbol{k}$ in $\mathfrak{S}$, $f(\boldsymbol{h}, \boldsymbol{k})=\boldsymbol{h} \cdot \boldsymbol{V} \cdot \boldsymbol{k}$. The operator $\boldsymbol{V}_{\mathfrak{H}}$ is called the variance of $\mu$, and is written heuristically as $\int_{\mathfrak{S}}\left(x-m_{\mathfrak{C}}\right)\left(x-m_{\mathfrak{H}}\right) \mu(d x)$. If $V_{\mathfrak{H C}}$ exists and $\mathfrak{T}$ is any finite dimensional subspace of $\mathfrak{S}$, then the variance tensor $V_{\mathfrak{I}}=\int_{\mathfrak{T}}\left(x-m_{\mathfrak{J}}\right)$ $\left(x-m_{\mathfrak{I}}\right) \mu_{\mathfrak{I}}(d x)$ of $\mu_{\mathfrak{I}}$ on $\mathfrak{T}$ exists, and $V_{\mathfrak{I}}=\mathfrak{J} \cdot V_{\mathfrak{T C}} \cdot \mathfrak{J}$.

The characteristic function $\phi_{\mu}: \mathfrak{S} \rightarrow C$ ( $C$ is the complex plane) of a cylinder measure $\mu$ on $\mathfrak{S}$ is defined for any $\boldsymbol{h}$ in $\mathfrak{S}$ by the equation $\phi_{\mu}(\boldsymbol{h})=\int_{\mathfrak{G}} e^{i \mathbf{h} \cdot \mathbf{x}} \mu(d x)$. 
Getoor $^{12}$ has shown that a function $\phi: \mathfrak{F} \rightarrow C$ is the characteristic function of a cylinder measure iff (if and only if) $\phi(o)=1$ and $\phi \mid \mathfrak{T}$ is continuous and positive definite for each finite-dimensional subspace $\mathfrak{T}$ of $\mathfrak{S}$, and that $\phi$ uniquely determines its cylinder measure.

Now suppose $\mathfrak{S}=\mathfrak{S} \dot{+} \mathfrak{T}$ with $\mathfrak{S}$ and $\mathfrak{T}$ possibly infinite dimensional. Suppose that $\mu_{\mathcal{S}}$ and $\mu_{\mathfrak{J}}$ are cylinder measures on $\mathfrak{S}$ and $\mathfrak{T}$. We claim that there is exactly one cylinder measure on $\mathfrak{S}$, which we call $\mu_{\mathcal{S}+\mathfrak{I}}$, with the following property: for any cylinder sets $S \subseteq \mathfrak{S}$ and $T \subseteq \mathfrak{I}$, if $U \subseteq \mathfrak{F}$ is the cylinder set $U=\left(S+\mathfrak{S}^{\perp}\right) \cap\left(T+\mathfrak{T}^{\perp}\right)$, then $\mu_{\mathcal{S}+\mathfrak{J}}(U)=\mu_{\mathcal{S}}(S) \mu_{\mathfrak{J}}(T)$. Uniqueness is clear, because any such cylinder measure must have the characteristic function $\phi_{\mathfrak{S}+\mathfrak{J}}(\boldsymbol{h})=\phi_{\mathfrak{S}}\left(\mathcal{S}_{\mathfrak{J}} \cdot \boldsymbol{h}\right)_{\phi_{\mathfrak{J}}}\left(J_{\mathfrak{S}} \cdot \boldsymbol{h}\right)$. But if we define a function $\phi_{\mathfrak{S}+\mathfrak{J}}: \mathfrak{S} \rightarrow C$ by this equation, $\phi_{S+J}$ satisfies the hypothesis of Getoor's theorem, and hence does come from a cylinder measure $\mu_{\mathcal{S}+\mathfrak{J}}$ on $\mathfrak{S}$. It is easy to check that $\mu_{\mathcal{S}+\Im}$ has the required

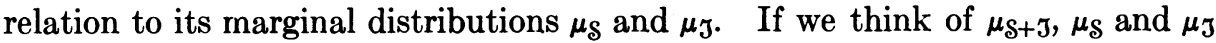
as probability distributions (even though they may not be countably additive), then we can think of $\boldsymbol{h}, \boldsymbol{S} \cdot \boldsymbol{h}$ and $\boldsymbol{J} \cdot \boldsymbol{h}$ as random variables, and $\boldsymbol{\mu}_{\mathcal{S}+\boldsymbol{J}}$ has been chosen so that $\boldsymbol{S} \cdot \boldsymbol{h}$ and $\mathfrak{J} \cdot \boldsymbol{h}$ are independently distributed ${ }^{11}$ on $\mathfrak{S}$.

Now suppose that $\mu_{\mathcal{S}}$ and $\mu_{\mathfrak{J}}$ have means $m_{\mathcal{S}}$ and $m_{\mathfrak{J}}$ and variances $V_{\mathcal{S}}$ and $V_{\mathfrak{J}}$. Then for any $h$ in $\mathfrak{S}, \int_{\mathfrak{G}} h \cdot x \mu_{\mathfrak{S}+\mathfrak{J}}(d x)=\int_{\mathfrak{S}} h \cdot\left(\mathfrak{S}_{\mathfrak{J}}^{*} \cdot \mathfrak{S} \cdot x+\mathfrak{J}_{\mathfrak{S}}^{*} \cdot \mathfrak{J} \cdot x\right)$ $\mu_{\delta+\Im}(d x)$. The integrand on the right is the sum of a cylinder function based on the subspace of $\subseteq$ spanned by $\delta_{\mathfrak{J}} \cdot \boldsymbol{h}$ and a cylinder function based on the subspace of $\mathfrak{I}$ spanned by $\mathfrak{J}_{\mathfrak{S}} \cdot \boldsymbol{h}$. Therefore $\int_{\mathfrak{S}} \boldsymbol{h} \cdot x_{\mu}(d x)=\int_{\Re} \boldsymbol{h} \cdot \delta_{\mathfrak{J}}{ }^{*} \cdot \boldsymbol{s} \mu_{\mathfrak{S}}(d s)+$ $\int_{\mathfrak{T}} \boldsymbol{h} \cdot J_{\mathfrak{S}}{ }^{*} \cdot \boldsymbol{t}_{\mathfrak{J}}(d t)$. The right hand side is $\boldsymbol{h} \cdot S_{\mathfrak{J}}{ }^{*} \cdot \mathrm{m}_{\mathcal{S}}+\boldsymbol{h} \cdot \mathfrak{J}_{\mathfrak{S}}^{*} \cdot \boldsymbol{m}_{\mathfrak{J}}$, so the

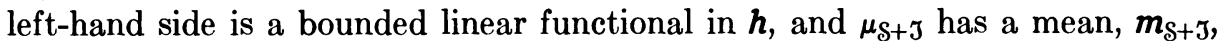
given by

$$
m_{S+J}=\varsigma_{\mathfrak{J}}^{*} \cdot m_{\mathfrak{S}}+J_{\mathfrak{S}}^{*} \cdot m_{\mathfrak{J}} .
$$

If for any $\boldsymbol{h}$ in $\mathfrak{S}$ we define $\boldsymbol{V}_{\mathcal{S}} \cdot \boldsymbol{h}=\boldsymbol{V}_{\mathcal{S}} \cdot \mathcal{S} \cdot \boldsymbol{h}$ and $\boldsymbol{V}_{\mathfrak{J}} \cdot \boldsymbol{h}=\boldsymbol{V}_{\mathfrak{J}} \cdot \mathfrak{J} \cdot \boldsymbol{h}$ then a similar argument shows that $\mu_{\mathcal{S}+\mathfrak{J}}$ has a variance $V_{\mathcal{S}+\mathfrak{J}}: \mathfrak{S} \rightarrow \mathfrak{S}$ and that

$$
V_{\mathfrak{S}+\mathfrak{J}}=\mathfrak{S}_{\mathfrak{J}}^{*} \cdot V_{\mathfrak{S}} \cdot \mathfrak{S}_{\mathfrak{J}}+\mathfrak{J}_{\mathfrak{S}}^{*} \cdot V_{\mathfrak{J}} \cdot \mathfrak{J}_{\mathfrak{S}} \text {. }
$$

Comparison with (5) then shows that

$$
V_{S+J^{i n v}}=V_{S}^{\text {inv }}+V_{J^{i n v}} \text {. }
$$

4. A Single Prediction from Error-free Data. As in $\mathrm{I}^{1}, d_{1}, \ldots, d_{D}$ are known vectors in the Hilbert space $\mathfrak{S}$ of models which contains the unknown correct model $\boldsymbol{h}_{E}$, and we suppose that $\gamma_{i}=\boldsymbol{d}_{i} \cdot \boldsymbol{h}_{E}$ has been measured exactly for $i=$ $1, \ldots, D$. Let $\mathfrak{D}$ be the closed subspace of $\mathfrak{S}$ spanned by $\boldsymbol{d}_{1}, \ldots, \boldsymbol{d}_{D}$. Then the data are equivalent to knowledge of the vector $D \cdot m_{E}=\sum_{i=1}^{D} \gamma_{i} d^{i}$, where $d^{1}, \ldots, d^{D}$ is the basis for $\mathfrak{D}$ dual to $d_{1}, \ldots, d_{D}$. Using the data, we want to estimate $\boldsymbol{p} \cdot \boldsymbol{h}_{E}$, where $\boldsymbol{p}$ is another known vector in $\mathfrak{S}$. The vectors $\boldsymbol{a}$ for which we can calculate $\boldsymbol{a} \cdot \boldsymbol{h}_{E}$ from the data are precisely the members of $\mathfrak{D}$. Therefore 
we might seek that vector $\boldsymbol{a}$ in $\mathfrak{D}$ which is most nearly equal to $p$ and estimate $\boldsymbol{p} \cdot \boldsymbol{h}_{\boldsymbol{E}}$ as $\boldsymbol{a} \cdot \boldsymbol{h}_{\boldsymbol{E}}$. The optimal $\boldsymbol{a}$ then minimizes $\|\boldsymbol{a}-\boldsymbol{p}\|$ under the constraint that $\boldsymbol{a}$ be in $\mathfrak{D}$; $\operatorname{thus}^{3} \boldsymbol{a}=\mathfrak{D} \cdot \boldsymbol{p}$, and we estimate $\boldsymbol{p} \cdot \boldsymbol{h}_{E}$ as $(\boldsymbol{p} \cdot \mathbb{D}) \cdot \boldsymbol{h}_{E}=\boldsymbol{p} \cdot\left(\mathfrak{D} \cdot \boldsymbol{h}_{E}\right)$. If we think it very likely that $\left\|\boldsymbol{h}_{E}\right\| \leq M$, then from Schwarz's inequality the maximum error in our estimate is $\|\boldsymbol{p}-D \cdot p\| M$.

In some investigations ${ }^{13}$ it is expedient to demand not only that $a$ be in $\mathfrak{D}$ but also that $\boldsymbol{a}$ treat $n$ given vectors $\boldsymbol{h}_{1}, \ldots, \boldsymbol{h}_{n}$ exactly; that is, $\boldsymbol{a} \cdot \boldsymbol{h}_{\nu}=\boldsymbol{p} \cdot \boldsymbol{h}_{\boldsymbol{\nu}}, \boldsymbol{\nu}=$ $1, \ldots, n$. Then we want to minimize $\|\boldsymbol{a}-\boldsymbol{p}\|$ subject to the demand that $\boldsymbol{a}$ be in $\mathfrak{D} \cap(\boldsymbol{p}+\mathfrak{F})$ where $\mathfrak{F}$ is the closed subspace of $\mathfrak{S}$ consisting of all vectors in $\mathfrak{S}$ orthogonal to $\left\{\boldsymbol{h}_{\mathbf{1}}, \ldots, \boldsymbol{h}_{n}\right\}$. If the problem is to be soluble, $\mathfrak{D} \cap(\boldsymbol{p}+\mathfrak{F})$ must be nonempty, so $\boldsymbol{p}$ must be in $\mathfrak{D}+\mathfrak{F}$. Therefore we lose no generality by assuming $\mathfrak{S}=\mathfrak{D}+\mathfrak{F}$. Let $\mathfrak{B}=\mathfrak{F} \ominus(\mathfrak{D} \cap \mathfrak{F})$. Then $\mathfrak{S}=\mathfrak{B}+\mathfrak{D}$ and, according to the penultimate paragraph of section 2 , the optimal $\boldsymbol{a}$ is $\mathbb{D}_{\mathbb{B}} \cdot \boldsymbol{p}$. Then we estimate $\boldsymbol{p} \cdot \boldsymbol{h}_{E}$ as $\left(\mathscr{D}_{\mathbb{B}} \cdot \boldsymbol{p}\right) \cdot \boldsymbol{h}_{E}=\boldsymbol{p} \cdot\left(\mathscr{D}_{\mathbb{B}}{ }^{*} \cdot \boldsymbol{h}_{E}\right)=\boldsymbol{p} \cdot\left(\mathscr{D}_{\mathbb{B}}{ }^{*} \cdot D_{D} \cdot \boldsymbol{h}_{E}\right)$. The error in the estimate is

$$
\left|\boldsymbol{p} \cdot \boldsymbol{h}_{E}-\left(D_{B} \cdot \boldsymbol{p}\right) \cdot \boldsymbol{h}_{E}\right|=\left|\left\langle\boldsymbol{p}-D_{B} \cdot \boldsymbol{p}, \mathfrak{F} \cdot \boldsymbol{h}_{E}\right\rangle\right| \leq\left\|\boldsymbol{p}-D_{B} \cdot \boldsymbol{p}\right\| \boldsymbol{M}
$$

if we think it likely that $\left\|\mathfrak{F} \cdot \boldsymbol{h}_{E}\right\| \leq M$. The advantage of constraining $\boldsymbol{a}$ to lie in $\mathfrak{D} \cap(\boldsymbol{p}+\mathfrak{F})$ is that then we can estimate our error from a bound merely on $\left\|\mathfrak{F} \cdot \boldsymbol{h}_{\boldsymbol{E}}\right\|$ rather than on $\left\|\boldsymbol{h}_{\boldsymbol{E}}\right\|$ itself.

5. A Single Prediction from Erroneous Data. In the foregoing section suppose that $\gamma_{i}=\boldsymbol{d}_{i} \cdot \boldsymbol{h}_{E}, i=1, \ldots, D$, have not been measured exactly but that the errors $\delta \gamma_{i}$ have a joint probability distribution with mean $o$ whose variance matrix $V_{i j}=E\left(\delta \gamma_{i} \delta \gamma_{j}\right)$ is known. ( $E$ means expected value.) Then

$$
h^{\prime}=\sum_{i=1}^{D} \gamma_{i} d^{i}
$$

is only an estimate for $D \cdot h_{E}$, and is in error by $\sum_{i=1}^{D}\left(\delta \gamma_{i}\right) d^{i}$. The variance tensor $\boldsymbol{V}$ for the distribution of $\boldsymbol{h}^{\prime}$ in $\mathfrak{D}$ is

$$
\boldsymbol{V}=E\left[\left(\boldsymbol{h}^{\prime}-\mathfrak{D} \cdot \boldsymbol{h}_{E}\right)\left(\boldsymbol{h}^{\prime}-D \cdot \boldsymbol{h}_{E}\right)\right]=\sum_{i, j=1}^{D} \mathrm{~V}_{i} \boldsymbol{d}^{i} \boldsymbol{d}^{j}
$$

For any $\boldsymbol{a}$ in $\mathfrak{D} \cap(\boldsymbol{p}+\mathfrak{F})$, if we estimate $\boldsymbol{p} \cdot \boldsymbol{h}_{\boldsymbol{E}}$ as $\boldsymbol{a} \cdot \boldsymbol{h}^{\prime}$ we commit an error $(\boldsymbol{a}-\boldsymbol{p}) \cdot \boldsymbol{h}_{E}+\boldsymbol{a} \cdot\left(\boldsymbol{h}^{\prime}-D \cdot D \cdot \boldsymbol{h}_{E}\right)$. The expected value of the square of this error is $\left|(\boldsymbol{a}-\boldsymbol{p}) \cdot \mathfrak{F} \cdot \boldsymbol{h}_{\boldsymbol{E}}\right|^{2}+\boldsymbol{a} \cdot \boldsymbol{V} \cdot \boldsymbol{a}$. If we think it likely that $\left\|\mathfrak{F} \cdot \boldsymbol{h}_{\boldsymbol{E}}\right\| \leq M$, then the maximum value which this expected square error can have is $\epsilon(a)^{2}=M^{2} \| \boldsymbol{a}-$ $\boldsymbol{p} \|^{2}+\boldsymbol{a} \cdot \boldsymbol{V} \cdot \boldsymbol{a}$. In this situation, we want to choose $\boldsymbol{a}$ in $\mathfrak{D} \cap(\boldsymbol{p}+\mathfrak{F})$ so as to minimize not $\|\boldsymbol{a}-\boldsymbol{p}\|$ but $\boldsymbol{\epsilon}(\boldsymbol{a})$. At the optimum $\boldsymbol{a}, \boldsymbol{\epsilon}(\boldsymbol{a})$ must be stationary under all variations $\delta \boldsymbol{a}$ in $\mathfrak{D} \cap \mathfrak{F}$. This enables us to show that if an optimal $a$ exists it must be

$$
\boldsymbol{a}_{0}=\mathscr{D}_{\mathbb{B}} \cdot \boldsymbol{p}-\left[(\mathscr{D} \wedge \mathcal{F}) \cdot\left(M^{2} \mathscr{D}+\boldsymbol{V}\right) \cdot(\mathscr{D} \wedge \mathcal{F})\right]^{\text {inv }} \cdot \boldsymbol{V} \cdot \mathscr{D}_{\mathbb{B}} \cdot \boldsymbol{P}
$$

where $\mathfrak{D} \wedge \mathcal{F}$ is the orthogonal projection operator from $\mathfrak{S}$ onto $\mathfrak{D} \cap \mathfrak{F}$. If $a$ is in $\mathfrak{D} \cap(\boldsymbol{p}+\mathfrak{F})$, a simple calculation shows that $\epsilon(\boldsymbol{a})^{2}=\epsilon\left(\boldsymbol{a}_{0}\right)^{2}+M^{2}\left\|\boldsymbol{a}-\boldsymbol{a}_{0}\right\|^{2}$ $+\left(a-a_{0}\right) \cdot V \cdot\left(a-a_{0}\right)$. Since $V$ is positive definite, the stationary point $a_{0}$ is indeed a minimum for $\epsilon(a)$ when $a$ is in $\mathfrak{D} \cap(p+\mathfrak{F})$. 
We estimate $\boldsymbol{p} \cdot \boldsymbol{h}_{\boldsymbol{E}}$ as $\boldsymbol{a}_{0} \cdot \boldsymbol{h}$, which is $\boldsymbol{p} \cdot \tilde{\boldsymbol{h}}_{\mathbb{B}+\mathcal{D}}$, where

$$
\tilde{h}_{\mathscr{B}+D}=\mathscr{D}_{\mathbb{B}}{ }^{*} \cdot \boldsymbol{h}^{\prime}-\mathscr{D}_{\mathbb{B}}{ }^{*} \cdot V \cdot\left[(D \wedge F) \cdot\left(M^{2} \mathscr{D}+V\right) \cdot(D \wedge F)\right]^{\text {inv }} \cdot \boldsymbol{h}^{\prime} .
$$

The calculations by Backus and Gilbert ${ }^{14}$ of tradeoff curves between absolute error $\boldsymbol{a} \cdot \boldsymbol{V} \cdot \boldsymbol{a}$ and resolving length (measured by $\|\boldsymbol{a}-\boldsymbol{p}\|)$ are a special case of (13) in which the dimension of $\mathfrak{F}^{\perp}$ is 1 . The Lagrange multiplier $\lambda$ which appears in those calculations is, in fact, $M^{2}$.

6. Several Predictions from Error-free Data. Suppose we want to estimate $\boldsymbol{p}_{1} \cdot \boldsymbol{h}_{E}, \ldots, \boldsymbol{p}_{\boldsymbol{P}} \cdot \boldsymbol{h}_{\boldsymbol{E}}$, given $\boldsymbol{p}_{1}, \ldots, \boldsymbol{p}_{P}, \mathbb{D} \cdot \boldsymbol{h}_{\boldsymbol{E}}$ and the hypothesis that $\left\|\mathfrak{F} \cdot \boldsymbol{h}_{\boldsymbol{E}}\right\| \leq M$. We could estimate each $\boldsymbol{p}_{\boldsymbol{v}} \cdot \boldsymbol{h}_{\boldsymbol{E}}$ separately and calculate the errors in our estimates as in section 4. Clearly, however, these errors cannot all reach their maximum size at once. What we would really like to know is the subset of $R^{P}$ to which the $P$-tuple $\left(\boldsymbol{p}_{1} \cdot \boldsymbol{h}_{E}, \ldots, \boldsymbol{p}_{P} \cdot \boldsymbol{h}_{E}\right)$ is confined by the data and the hypothesis $\left\|\mathfrak{F} \cdot h_{E}\right\| \leq M$. Let $\mathfrak{B}$ be the subspace of $\mathfrak{S}$ spanned by $p_{1}, \ldots, p_{P}$. Then we want to know what limits are placed on $\mathcal{P} \cdot \boldsymbol{h}_{E}$ by a knowledge of $\mathscr{D} \cdot \boldsymbol{h}_{E}$ and the assumption $\left\|\mathfrak{F} \cdot h_{E}\right\| \leq M$. That is, we would like to know $S(\mathfrak{D}, \mathfrak{F}, M)$, the set of all vectors $\boldsymbol{P} \cdot \boldsymbol{h}$ such that $\boldsymbol{h}$ is in $\mathfrak{S}, \mathfrak{D} \cdot \boldsymbol{h}=\mathfrak{D} \cdot \boldsymbol{h}_{\boldsymbol{E}}$, and $\|\mathfrak{F} \cdot \boldsymbol{h}\| \leq M$. If $S(\mathfrak{D}, \mathfrak{F}$,$M)$ is to be bounded, we must have $\mathfrak{B} \subseteq \mathfrak{D}+\mathfrak{F}$; we will assume this, and therefore lose no generality in assuming $\mathfrak{S}=\mathfrak{D}+\mathfrak{F}$.

It is easy to verity that the solution to this problem in solid geometry is

$$
\begin{gathered}
S(\mathfrak{D}, \mathfrak{F}, M)=P \cdot \mathscr{D}_{\mathbb{B}}{ }^{*} \cdot \boldsymbol{h}_{E}+\{\boldsymbol{n}: \boldsymbol{n} \text { in } \mathfrak{N} \text { and } \\
\left.\boldsymbol{n} \cdot \mathfrak{T} \cdot \mathscr{B}_{\mathbb{D}}{ }^{*} \cdot \mathscr{B}_{\mathfrak{D}} \cdot \mathfrak{T} \cdot \boldsymbol{n} \leq M^{2}-\left\|\mathscr{D} \wedge \mathfrak{F} \boldsymbol{h}_{E}\right\|^{2}\right\},
\end{gathered}
$$

where $\mathfrak{B}=\mathfrak{F} \ominus(\mathfrak{D} \cap \mathfrak{F})$ and $\mathfrak{R}=\mathfrak{B} \ominus(\mathfrak{D} \cap \mathfrak{P})$. Thus $S(\mathfrak{D}, \mathfrak{F}, M)$ is a nondegenerate ellipsoid in $\mathcal{P} \cdot \mathscr{D}_{\mathbb{B}}{ }^{*} \cdot h_{E}+\mathfrak{R}$, whose principal axes are determined by the eigenvectors and eigenvalues of $\mathscr{T} \cdot \mathbb{B}_{\mathfrak{D}}^{*} \cdot B_{\mathfrak{D}} \cdot \mathfrak{K}$.

7. Several Predictions from Erroneous Data. We continue to use the notation of sections 5 and 6 . If $P>1$ and the data $\gamma_{i}$ are inaccurate, we do not know how to generalize efficiently and linearly the approach of section 5 . Therefore we adopt a Bayesian point of view. Our belief that $\left\|\mathfrak{F} \cdot \boldsymbol{h}_{\boldsymbol{E}}\right\|$ is probably not much larger than $M$ can be embodied in an a priori subjective probability distribution $^{15}$ of $\mathfrak{F} \cdot \boldsymbol{h}_{E}$ on $\mathfrak{F}$. This distribution must be a cylinder measure $\boldsymbol{\mu}_{\mathfrak{F}}$ rather than a countably additive probability measure if $\mathfrak{F}$ is infinite-dimensional. A convenient and natural choice of $\mu_{\mathcal{F}}$ is the cylinder measure whose characteristic function is $\phi_{\mathcal{F}}(f)=\exp \left[-M^{2}\|f\|^{2} / 2\right]$. On any finite-dimensional subspace $\mathfrak{T}$ of $\mathfrak{F}$, the marginal distribution of $\mu_{\mathfrak{F}}$ is normal with mean $\boldsymbol{O}$ and variance tensor $M^{2} \mathfrak{J}$, so $\mu_{\mathcal{F}}$ has mean $o$ and variance tensor $M^{2} \mathcal{F}$. The marginal distribution of $\mu_{\mathfrak{F}}$ on $\mathfrak{D} \cap \mathfrak{F}$ has probability density $p_{\text {prej }}$ given by $-2 \ln p_{\text {prej }}(D \wedge F \cdot h)$ $=$ constant $+M^{-2}\|D \wedge \mathcal{F} \cdot \boldsymbol{h}\|^{2}$.

The distribution of errors in the data can be thought of as a probability distribution on $\mathfrak{D}$ with density $p_{\text {data }}$, mean $\boldsymbol{h}^{\prime}$ and variance tensor $V$ given by (10) and (11). The Bayesian approach ${ }^{15}$ leads us to adopt an $a$ posteriori subjective probability distribution for $\mathfrak{D} \cdot \boldsymbol{h}$ in $\mathfrak{D}$, whose probability density is $\boldsymbol{p}_{\mathfrak{D}}$ with $\boldsymbol{p}_{\mathfrak{D}}$ $(D \cdot h)=K p_{\text {data }}(D \cdot h) p_{\text {prej }}(D \wedge F \cdot h)$, where $K$ is a normalizing constant. As in I, this $\boldsymbol{p}_{\mathfrak{D}}$ uses our prejudice about $\left\|\mathfrak{F} \cdot \boldsymbol{h}_{\boldsymbol{E}}\right\|$ to limit the variance of $D \cdot \boldsymbol{h}_{\boldsymbol{E}}$ in those directions in $\mathfrak{D} \cap \mathfrak{F}$ for which the data provide no useful limit. 
If $p_{\text {data }}$ is normal then $p_{\mathscr{D}}$ is normal, with variance tensor $V_{\mathscr{D}}$ and mean $\boldsymbol{h}_{\mathfrak{D}}$ given by

$$
\begin{gathered}
V_{\mathscr{D}}^{\mathrm{inv}}=V^{\mathrm{inv}}+M^{-2} \mathscr{D} \wedge \mathcal{F} \\
\boldsymbol{h}_{\mathscr{D}}=V_{\mathscr{D}} \cdot \boldsymbol{V}^{\mathrm{inv}} \cdot \boldsymbol{h}^{\prime} .
\end{gathered}
$$

Since $\mathfrak{B} \cap \mathfrak{D}=\{\boldsymbol{o}\}$, the only limitation on $\mathbb{B} \cdot \boldsymbol{h}_{\boldsymbol{E}}$ comes from our subjective probability $\mu_{\mathcal{F}}$, which gives for the distribution of $\mathbb{B} \cdot \boldsymbol{h}_{E}$ on $\mathfrak{B}$ the cylinder measure $\mu_{\mathscr{B}}$, whose characteristic function is $\phi_{\mathscr{B}}=\phi_{\mathfrak{F}} \mid \mathfrak{B}$. The mean and variance

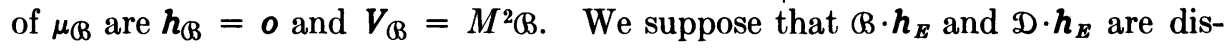
tributed independently and that $\mu_{\mathbb{B}}$ and $\mu_{D}$ are the marginal distributions of a

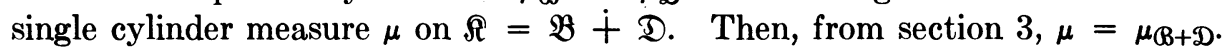
(This conclusion can also be reached by a Bayesian argument.) The net effect of the data and our prejudice is to produce for us an a posteriori subjective probability distribution, $\mu_{\mathscr{B}+D}$, for $\mathscr{K} \cdot \boldsymbol{h}$ in $\Re$. The mean $\boldsymbol{h}_{\mathscr{B}+\mathbb{D}}$ and variance $\boldsymbol{V}_{\mathbb{B}+\mathscr{D}}$ are given by (see (7) and (9))

$$
\begin{gathered}
h_{\mathscr{B}+\mathscr{D}}=\mathscr{D}_{\mathbb{B}}{ }^{*} \cdot V_{\mathscr{D}} \cdot V^{\mathrm{inv}} \cdot \boldsymbol{h}^{\prime} \\
V_{\mathscr{B}+D^{\mathrm{inv}}}=V_{\mathscr{D}}^{\mathrm{inv}}+V_{\mathscr{B}}{ }^{\mathrm{inv}} .
\end{gathered}
$$

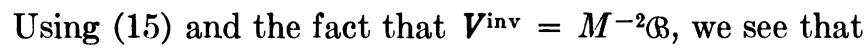

$$
V_{\mathbb{B}+D^{\text {inv }}}=V^{\text {inv }}+M^{-2} \mathcal{F} \text {. }
$$

Unless $\mathfrak{B} \subseteq \mathfrak{B}+\mathfrak{D}$ there is no marginal distribution of $\mu_{\mathfrak{B}+\mathbb{D}}$ on $\mathfrak{B}$ and no way to make our predictions. Therefore we assume $\mathfrak{B} \subseteq \mathfrak{B}+\mathfrak{D}=\mathfrak{D}+\mathfrak{F}$. Then we lose no generality by assuming that $\mathfrak{S}=\mathfrak{D}+\mathfrak{F}$. With this assumption, $\mu_{\circledast+D}$ is a cylinder measure on $\mathfrak{S}$, and the prediction vector $\boldsymbol{P} \cdot \boldsymbol{h}_{\boldsymbol{E}}$ has an a posteriori subjective probability distribution with mean $\boldsymbol{h}_{\mathcal{P}}=\boldsymbol{P} \cdot \boldsymbol{h}_{\mathbb{B}+\mathbb{D}}$ and variance tensor $V_{\mathcal{P}}=\boldsymbol{P} \cdot \boldsymbol{V}_{\mathbb{B}+\mathcal{D}} \cdot \boldsymbol{P}$. The $P$-tuple of predictions $\left(\boldsymbol{p}_{1} \cdot \boldsymbol{h}_{E}, \ldots, \boldsymbol{p}_{P}\right.$. $\boldsymbol{h}_{\boldsymbol{E}}$ ) has a probability distribution in $R^{\boldsymbol{P}}$ whose mean is $\left(\boldsymbol{p}_{1} \cdot \boldsymbol{h}_{\odot}, \ldots, \boldsymbol{p}_{P} \cdot \boldsymbol{h}_{\odot}\right)$ and whose variance matrix is $E\left[\boldsymbol{p}_{i} \cdot\left(\boldsymbol{h}_{E}-\boldsymbol{h}_{\odot}\right)\left(\boldsymbol{h}_{E}-\boldsymbol{h}_{\odot}\right) \cdot \boldsymbol{p}_{j}\right]=\boldsymbol{p}_{i} \cdot \boldsymbol{V}_{\mathcal{P}} \cdot \boldsymbol{p}_{j}$.

For readers skeptical of Bayes it will be of interest to note that when the foregoing Bayesian calculus is applied to a single prediction, the result is the same as that obtained in the objectivist, non-Bayesian section 5 . We want to show from

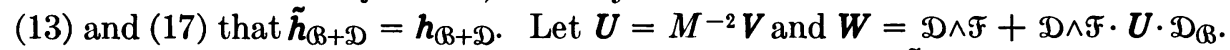
Then $W$ lives on $\mathfrak{D} \cap \mathfrak{F}$ and $U$ lives on $\mathfrak{D}$. We have $\tilde{h}_{\mathbb{B}+\mathbb{D}}=\mathscr{D}_{\mathbb{B}}{ }^{*} \cdot(\mathscr{D}-$ $\left.\boldsymbol{U} \cdot \boldsymbol{W}^{\mathrm{inv}}\right) \cdot \boldsymbol{h}^{\prime}$ and $\boldsymbol{h}_{\mathbb{B}+\mathbb{D}}=\mathbb{D}_{\mathbb{B}}{ }^{*} \cdot(\mathbb{D}+\boldsymbol{U} \cdot \mathbb{D} \wedge \mathcal{F})^{\mathrm{inv}} \cdot \boldsymbol{h}^{\prime}$. But $\mathbb{D}+\boldsymbol{U} \cdot \mathbb{D} \wedge \mathcal{F}=\mathfrak{C}$ $+\mathcal{C} \cdot U \cdot D \wedge \mathcal{F}+W$, so, from $(5),(D+U \cdot D \wedge F)^{\text {inv }}=\mathfrak{e}-\mathfrak{e} \cdot U \cdot W^{\text {inv }}+W^{\text {inv }}$ $=\mathfrak{D}-\boldsymbol{U} \cdot \boldsymbol{W}^{\text {inv. }}$ Here $\mathfrak{C}=\mathfrak{D} \ominus(\mathfrak{D} \cap \mathfrak{F})$.

* Supported in part by the Geophysics section of the National Science Foundation.

1 Backus, G., Proc. Nat. Acad. Sci. USA, 65, 1 (1970).

2 Backus, G., Proc. Nat. Acad. Sci. USA, 65, 281 (1970).

${ }^{3}$ Halmos, P. R., Introduction to Hilbert Space (New York: Chelsea, 1951).

4 Moore, E. H., Bull. Amer. Math. Soc., 26, 393 (1920).

- Penrose, R., Proc. Cambridge Phil. Soc., 51, 406 (1955).

- Lorch, E. R., Trans. Amer. Math. Soc., 45, 217 (1939).

7 Dunford, N., and J. T. Schwartz, Linear Operators I (New York: Interscience, 1958).

8 Gross, L., Analysis in Function Space, ed. W. T. Martin and I. E. Segal (Cambridge, Mass.: M. I. T. Press, 1964), pp. 51-68. 
- Halmos, P. R., Measure Theory (New York: Van Nostrand, 1950).

10 Segal, I. E., Trans. Amer. Math. Soc., 81, 106 (1956).

11 Parzen, E., Modern Probability Theory and its Applications (New York: Wiley, 1960).

12 Getoor, R. K., Pacific J. Math., 7, 885 (1957).

12 Backus, G., and F. Gilbert, Geophys. J. Roy. Astron. Soc., 16, 169 (1968).

14 Backus, G., and F. Gilbert, Phil. Trans. Roy. Soc. London Ser. A, 266, 123 (1970).

16 Savage, L. J., The Foundations of Statistical Inference (London: Methuen, 1962), p. 22. 